\title{
Positron Annihilation Lifetime Analysis of Left- and Right-Handed Alanine Single Crystals
}

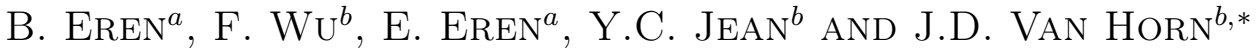 \\ ${ }^{a}$ Department of Chemistry, Faculty of Science and Arts, Bilecik Şeyh Edebali University, 11210 Bilecik, Turkey \\ ${ }^{b}$ Department of Chemistry, University of Missouri-Kansas City, \\ 5110 Rockhill Rd., Kansas City, Missouri 64110, USA
}

\begin{abstract}
Studies of the interaction of polarized light or particles (including electrons, $\mathrm{e}^{-}$, or positrons, $\mathrm{e}^{+}$) with asymmetric forms of matter has been of interest to scientists since the discovery of chirality and the subsequent development of particle physics. Researchers have been interested in $\mathrm{e}^{+}$interactions with chiral molecules for decades, but with mixed and indecisive results. After reviewing the field, we speculated that the $\mathrm{e}^{+}$or positronium $(\mathrm{Ps}) \mathrm{might}$ interact differently with chiral pairs of large single crystals, i.e., the left-handed or right-handed asymmetric forms of the crystals - and subsequently observed significant differences in "free positron" annihilation and intensities in the evaluation of left-handed or right-handed quartz single crystals. This result may be understood to be a "particle stereorecognition" phenomenon. To extend this line of inquiry we crystallized mm scale L- or D-alanine crystals and performed positron annihilation lifetime spectroscopy measurements using a ${ }^{22} \mathrm{Na}$ positron source. Alanine crystals were obtained via slow evaporation of water in a Dewar, or from water/acetone solvent in a temperature-controlled environment. These methods resulted in small $(\approx 0.5 \mathrm{~cm} /$ side $)$ or large $(\geq 1.0 \mathrm{~cm} /$ side $)$ crystals, respectively. While some intensity $\left(I_{2}\right)$ results from left-handed and right-handed crystals varied in positron annihilation lifetime spectroscopy analysis, the errors associated with the measurements do not indicate a stereorecognition of alanine via positron interactions.
\end{abstract}

DOI: 10.12693/APhysPolA.132.1456

PACS/topics: 78.70.Bj, 82.30.Gg, 34.80.-i, 87.15.B-

\section{Introduction}

Asymmetry and the interactions of asymmetric entities are particularly important phenomena in the universe. One could say that: "Asymmetry is more important than symmetry"(J.D.V.H.), especially in the realm of biochemical interactions. The origin of biological asymmetry and a mechanistic connection between the asymmetric nuclear weak force and molecular asymmetry (i.e. chirality) remain outstanding scientific problems [1-4].

The history of positron experiments with asymmetric matter caught our attention as being a significant research area despite small, ambiguous, or negative results observed over the last four decades [5-11]. This interest extends to the other beta particle, the electron, and polarized electron experiments with asymmetric matter that have been recently appearing in the literature [1214]. Of particular interest were positron annihilation experiments with chiral liquids and their frozen form [5] and experiments with amino acid powders to determine whether positrons could selectively destroy one amino acid enantiomeric form over the other [8-10]. The results in these experiments again have been mostly negative, but additional experiments continue to be performed, including a gas phase positron scattering experiment with chiral ester molecules in the gas phase [11]. The history and a survey of positron experiments with asymmetric

\footnotetext{
*corresponding author; e-mail: vanhornj@umkc.edu
}

matter will be the subject of a separate review article, currently in progress.

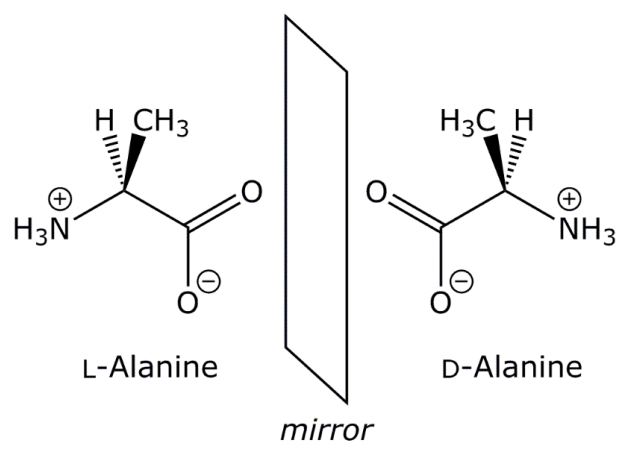

Fig. 1. Molecular structures of L- and D-alanine.

In this paper, we briefly develop a classification scheme for possible asymmetric interactions between polarized particles and molecular entities. Second, an experimental probe of positron interactions with asymmetric single crystals, specifically the amino acid alanine (Fig. 1) is described. The result here may be also considered a followup study and extension of early work by Garay et al. [6], Rich [8], and others investigating positron annihilation in L- and D-amino acids over the years.

\section{Experimental}

Alanine crystals (Fig. 2) were obtained from solvent crystallization of enantiomerically pure L- or D-amino 


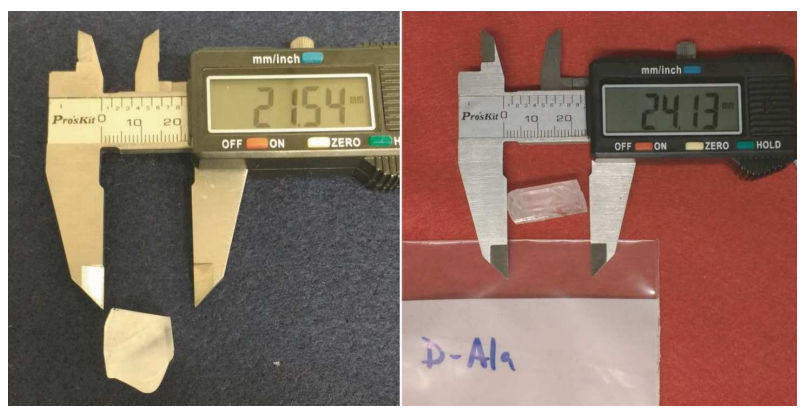

Fig. 2. Examples of crystals of L- or D-alanine grown from an acetone/water solvent system.

acid powders (L-alanine, 99+\%, and D-alanine, 99+\%, ACROS Organics $\left.{ }^{\mathrm{TM}}\right)[15,16]$. The term "asymmetry" is used interchangeably with "chirality" or "helicity" depending on context; RH (right-handed) or LH (lefthanded) are used interchangeably for the amino acid designations L- or D-, respectively. Acetone was obtained from ACROS Organics ${ }^{\top M}$ (99.6\%, for spectroscopy) and in-house doubly deionized water $(>18 \mathrm{M} \Omega$ ) was used in all preparations. Large crystals (approximately $1 \times 1 \times 2 \mathrm{~cm}^{3}$ ) were obtained by the slow evaporation (days to weeks) of solvent in an acetone/water/alanine system in a controlled temperature environment at $25^{\circ} \mathrm{C}$. In a separate method, alanine crystals were obtained directly from the slow cooling (weeks) of saturated water/alanine solutions. A warm alanine solution $\left(60^{\circ} \mathrm{C}\right)$ in a plastic tube was placed in a Dewar filled with warm water as a thermal mass $\left(60^{\circ} \mathrm{C}\right)$ and the Dewar/water/solution system was slowly cooled to $30^{\circ} \mathrm{C}$; subsequently, the solution was maintained at temperature by placing it in a $30^{\circ} \mathrm{C}$ oven. Typically, the formation of crystals was slow and seed crystals from previous, shorter crystallization batches were added to new solutions to develop larger crystals for positron annihilation lifetime spectroscopy (PALS) analysis. Crystals were carefully dried and optically inspected for flaws. Suitable crystals were selected and carefully fractured to give two pieces; the pieces from the same crystal were used in a sample sandwich for positron analysis. The positron source was placed either between the crystal pieces in the fracture or in a "back-to-back" fashion between flat crystal planes. In some experiments, two small crystals from a single crystallization batch were placed face-to-face for analysis. In a separate set of experiments, positron analysis was completed on samples before and after the polishing of crystal faces of interest. This "polishing" was accomplished by a single pass of the face of each crystal on a 600 grit paper followed by gentle wiping with a lint-free tissue.

Positron spectrometric measurements were taken using a conventional fast-fast coincidence method with events compiled with a multichannel analyzer as previously described [17-19]. The time resolution of the spectrometer was $<300 \mathrm{ps}$, measured in a $100 \mathrm{~ns}$ window using a ${ }^{60} \mathrm{Co}$ source with ${ }^{22} \mathrm{Na}$ energy settings $[20] . \quad \mathrm{A}{ }^{22} \mathrm{NaCl}$ positron source $(c a .70 \mu \mathrm{Ci}$, sealed in Kapton film) was sandwiched between two pieces (all edges $>0.5 \mathrm{~cm}$ dimension) of crystal as detailed above. PAL spectra (4 million counts in a region of interest) were collected, using a $100 \mathrm{~ns}$ window for each sample at ambient conditions $\left(25^{\circ} \mathrm{C}\right)$, but sealed in an airtight bag, using a system based on ORTEC ${ }^{\circledR}$ positron lifetime systems (AMETEK, Inc., Oak Ridge, TN). Positron interaction with the source coating was accounted for in the data analysis (10\%). All PAL spectra were resolved into three components using the PATFIT program [21]. Spectra were also treated with the MELT routine [22], but without significantly different results.

\section{Results and discussion}

\subsection{Classification of beta particle interactions with asymmetric matter}

In comparing recent positron and electron experiments with asymmetric matter, we realized that these interactions could be classified in terms of their stereochemical outcome or intended result. Also, considering the physical state and chemistry of molecular entities [23] and by applying analogous stereochemical reactions concepts we classify asymmetric interactions in one of three ways: stereorecognition, stereoselection, and stereoinduction (emphasis added).

First, stereorecognition may be considered in any technique that can observe and distinguish the two asymmetric forms of some molecular entity or object. By way of example, optical polarimetry methods can distinguish solutions of chiral molecules or macroscopic chiral crystals. For a particle/molecule experiment that would represent stereorecognition, we would consider the scattering experiment by Chiari et al. [11] to be typical example. This experiment represents stereorecognition since it attempted to observe differential scattering between the left- and right-handed forms of molecules separately investigated in the gas phase.

The second category, stereoselection, may be applied to techniques that can selectively separate or interact (react) with one of a pair of asymmetric entities. A commonly used example in chemistry is that of liquid chromatography where an asymmetric (chiral) column packing material is used to separate asymmetric molecules via the interactions between the packed column and the solution-phase molecule. For a particle example of stereoselection (and stereorecognition) the recent work by Dreiling et al. is of great importance [12-14]. In these experiments, oppositely polarized electrons selectively interact with one asymmetric form of a selected chiral gasphase molecule. The researchers demonstrated that the intensity of a dissociative electron attachment reaction depended on the sign of the electron helicity; also observed was a difference in the magnitude of the asymmetry depending on a different heavy atom in the chiral molecule used in the experiment. Additionally, as mentioned in the introduction, studies of positron interaction 
with amino acid powders, and the selective radiolysis of one form over the other to lead to a chiral enhancement is a type of a stereoselective experiment.

Finally, stereoinduction would be the creation of asymmetry in a molecular system from some asymmetric process or force. Examples of this include the use of asymmetric catalysts to generate one specific asymmetric form of a molecule in preference to the other. Multiple examples of molecular asymmetric induction are known, but these arise from using asymmetric catalysts, hosts or templates. Presently, with respect to helical particle physics, it is difficult to conceive of a particle/molecule interaction that would transfer particle helicity (asymmetry) to give rise to an asymmetric result. However, one may speculate about molecular entities or structures that might be amenable to an induced asymmetry, depending on conditions and source of asymmetric particle.

Another concept related to asymmetric molecular interactions, and part of the motivation to re-investigate this aspect of positron chemistry, is that of "host-guest" interactions between asymmetric entities; these are commonplace in biological systems, supramolecular chemistry, and catalysis. Fundamentally, asymmetric hostguest interactions may be understood as arising from energy differences in the interaction of the two asymmetric forms of a guest with a particular host. That is, one of the interactions, "G ${ }_{L H} /$ Host" or "G $R H$ /Host", is more stable, thus preferentially forms; this may be also described as a preferential "diastereotopic" interaction. With this in mind, we envisaged that a helical positron could be considered to be a guest in materials; given the correct host, we should observe some type of positron annihilation phenomena as a result of the an asymmetric interaction. Positrons from ${ }^{22} \mathrm{Na}$ are ideally suited for this experiment both in beam or in bulk experiments since the beta radiation is largely polarized [24, 25].

Inspired by these classifications, the physical chemistry of chiral molecular systems and their interactions, and the characteristics of positrons and their annihilation, we envision two main types of possible positron interactions with asymmetric materials: stereorecognition and stereoselection.

For an initial test of these hypotheses, we selected leftand right-handed forms of quartz single crystals and observed free positron lifetime $\left(\tau_{2}\right)$ and intensity $\left(I_{2}\right)$ differences in PALS experiments with the asymmetric forms of these crystals [26]. We consider this result to be an example of "particle stereorecognition", as described above. After repeating this experiment with both natural and synthetic LH and RH quartz crystals, we turned our attention to "softer", asymmetric molecular crystals that could be related to organic or biological chemistry. The chiral molecules of interest include amino acids, carboxylic acids and esters, helicenes, and other crystallizable asymmetric organic molecules.

\subsection{PALS of left- or right-handed alanine crystals}

PAL spectra of alanine were collected using suitable crystals grown from either one of two crystallization methods. The first method yielded small crystals grown from water of dimensions approximating a half of centimeter per edge. A second method of crystallization from acetone/water solvent systems yielded larger crystals $(>1 \mathrm{~cm} /$ dimension). In most cases, the crystals were carefully cut or fractured to provide two pieces of the same crystal for positron analysis. Some experiments were performed using two crystals obtained from the same crystallization solution. The obtained positron annihilation spectra were fitted to three lifetime parameters and their associated intensities.

\section{TABLE I}

PALS results for L- or D-alanine crystals grown in water and acetone/water solution. Calculated errors in the last $\operatorname{digit}(\mathrm{s})$ are in round brackets.

\begin{tabular}{c|c|c|c|c}
\hline \hline & L-alanine & D-alanine & L-alanine & D-alanine \\
\cline { 2 - 5 } & \multicolumn{2}{|c}{ water } & \multicolumn{2}{c}{ acetone/water } \\
\hline$\tau_{1}[\mathrm{~ns}]$ & $0.230(06)$ & $0.231(12)$ & $0.170(5)$ & $0.213(6)$ \\
$\tau_{2}[\mathrm{~ns}]$ & $0.489(07)$ & $0.472(18)$ & $0.418(6)$ & $0.420(8)$ \\
$\tau_{3}[\mathrm{~ns}]$ & $1.390(63)$ & $1.250(85)$ & $1.31(5)$ & $1.68(7)$ \\
$I_{1}[\%]$ & $38.8(1.9)$ & $42.7(4.7)$ & $33.3(1.5)$ & $47.3(2.8)$ \\
$I_{2}[\%]$ & $58.6(1.6)$ & $53.4(4.0)$ & $63.5(1.3)$ & $50.7(2.7)$ \\
$I_{3}[\%]$ & $2.5(0.3)$ & $3.8(0.7)$ & $3.1(0.3)$ & $1.9(0.2)$ \\
$\tau_{1,2 a v .}[\mathrm{ns}]$ & $0.376(17)$ & $0.351(17)$ & $0.322(10)$ & $0.314(10)$
\end{tabular}

TABLE II

Repeated PALS experiments with L- or D-alanine crystals grown from water, before $(-)$ and after $(+)$ polishing the crystal faces used in the sample sandwich. Calculated errors in the last digit(s) are in round brackets; variance of fit was less than $1.0 \pm 0.08$.

\begin{tabular}{l|l|l|l|l|l|l}
\hline \hline & $\tau_{1}[\mathrm{~ns}]$ & $\tau_{2}[\mathrm{~ns}]$ & $\tau_{3}[\mathrm{~ns}]$ & $I_{1}[\%]$ & $I_{2}[\%]$ & $I_{3}[\%]$ \\
\hline \multicolumn{7}{c}{ L-alanine } \\
\hline$(-)$ & $0.250(6)$ & $0.526(12)$ & $1.49(11)$ & $49.2(2.4)$ & $48.7(2.1)$ & $2.1(0.4)$ \\
$(+)$ & $0.229(6)$ & $0.496(14)$ & $1.15(11)$ & $44.6(2.6)$ & $52.5(1.9)$ & $2.9(0.9)$ \\
\hline \multicolumn{7}{c}{ D-alanine } \\
\hline$(-)$ & $0.229(7)$ & $0.476(12)$ & $1.31(06)$ & $42.6(2.8)$ & $53.3(2.4)$ & $4.1(0.5)$ \\
$(+)$ & $0.217(6)$ & $0.478(11)$ & $1.21(06)$ & $40.6(2.4)$ & $55.4(1.9)$ & $4.0(0.6)$
\end{tabular}

Results for the small alanine crystals grown from water are listed in Table I (left). Employing a two lifetime component model for the resulting spectra led to larger calculated errors and poor fitting of observed and calculated curves. Thus, all spectra were subsequently fitted to three lifetime components, with good result. We hypothesize that the third lifetime component is associated with defects in the crystalline structure, and that the second lifetime component is associated with positron interactions with the lattice of the material, i.e. "free positron" annihilation. There is good agreement between the observed lifetimes between both LH and RH crystals. There appears to be some variation in $I_{1}$ and $I_{2}$ intensities, but averaging the first and second lifetime components $\left(\tau_{1,2 a v}\right.$, last row [27], Table I (left)) indicates that the differences in the intensity values between the left 
and right crystals is not significant. The observed $\tau_{3}$ and $I_{3}$ values are small, but have comparable values between the $\mathrm{LH}$ and $\mathrm{RH}$ crystals. The values suggest that $\tau_{3}$ may be associated with crystal packing defects, in contrast to typical values for polymer free volume defects at about $2.00 \mathrm{~ns}$. The small and similar values for $I_{3}$ suggest that the crystals are of good quality and have a low defect concentration. Comparable values for quartz crystals are $\tau_{3}=1.3-1.6 \mathrm{~ns}$ and $I_{3}=3.5-7.7 \%[26]$.

The positron annihilation results from large alanine crystals, grown in an acetone/water solvent system are presented in Table I (right). The results are comparable to those above, with a few differences. In this set of crystals, there is a large difference in the $\tau_{1}$ values, along with the previously noted $I_{1}$ and $I_{2}$ differences. Again, averaging the first and second lifetime components indicates a non-different result between the two samples within the attendant error. The results for the third lifetime component is similar to that of the water-grown crystals, indicating that both methods lead to experimentally suitable crystals with low defect density.

Because of the origin of the crystals, and the possibility of surface roughness or contamination, we were concerned about the interaction of the impinging positrons on the surfaces of the grown crystals. Thus, we collected PALS data with the small water-grown crystals before and after a surface treatment of the crystal. As noted in the experimental section, two suitable crystals were selected and analyzed using the best visually identified faces. Subsequently, the same crystals were "polished" on the same analysis surface to evaluate whether surface differences would lead to a significantly different result. The results of these studies are presented in Table II where the two asymmetric crystals are compared to each other, before and after the surface polishing. This set of data replicates those in the above experiments, but with a different set of LH or RH alanine crystals grown from water. Actually, this data presents a fuller view of the whole project, illustrating quite comparable values within the same crystal sample set and between left- and right-handed crystal samples. It is noteworthy that the observed lifetimes after treatment are generally lower than before the surface treatment. Intensity values exhibit mixed trends with $I_{1}$ values decreasing after polishing, but $I_{2}$ and $I_{3}$ values generally increase or remain the same after surface treatment. The observed $I_{3}$ values may provide some sense of crystal quality, assuming that $I_{3}$ provides some measure of crystal defect concentration. Comparing all data, the $I_{3}$ values for L-alanine are relatively consistent but vary somewhat over all the measured samples, ranging from 2.1 to $3.1 \%$. The D-alanine $I_{3}$ results are very comparable between the sample crystals grown from water and polished (3.8-4.1\%, compare Tables I and II), but is significantly lower $(2.1 \%)$ for crystals grown from acetone/water. While these results show some variability and we expect some amount of defect in the crystals as grown, we do not think that this is an indicator of poor crystal quality, given the results here taken together, and in comparison to $I_{3}$ values observed in synthetic single crystal quartz [26].
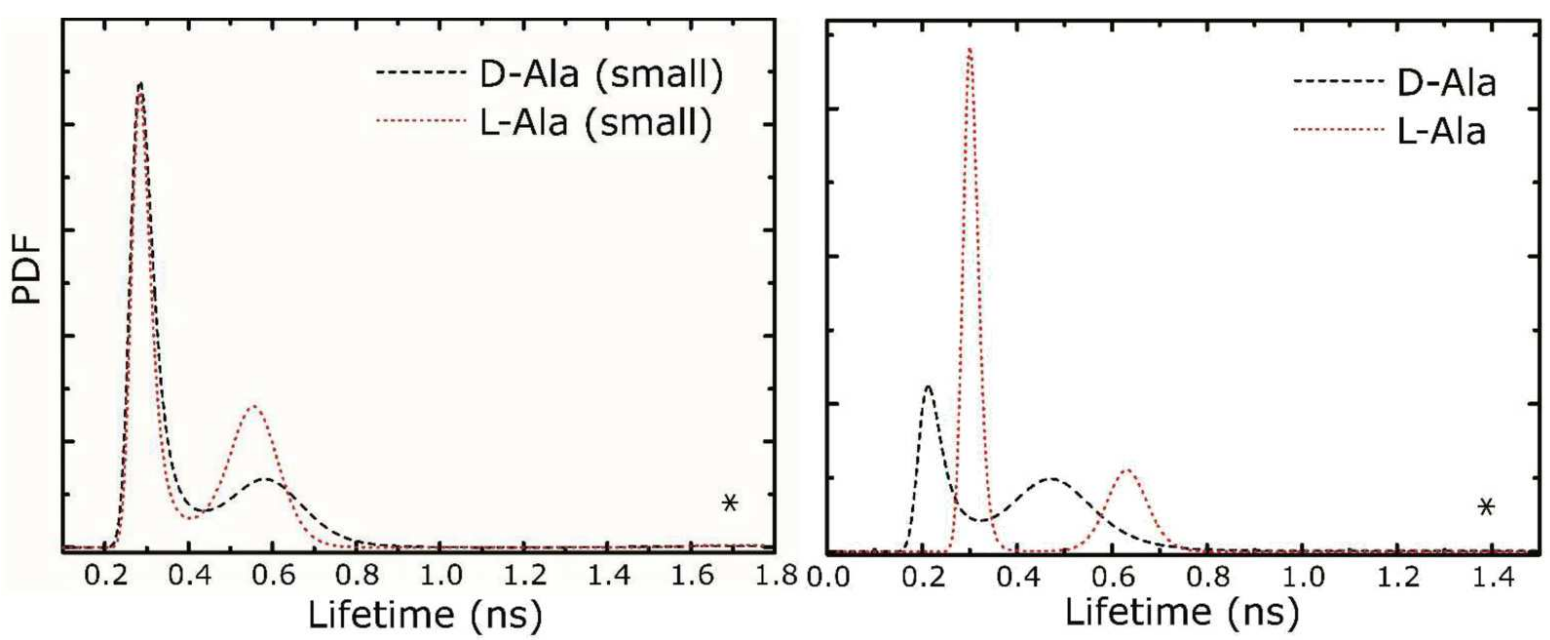

Fig. 3. Sample maximum entropy lifetime (MELT) results for L- and D-alanine crystals. See text for description and note the scale difference $\left({ }^{*}\right)$. PDF is a probability distribution function.

Finally, PALS data were evaluated with the statistical maximum entropy lifetime (MELT) method [22]. In the analysis, the maximum entropy value was set at $10^{-7}$, and resulting average entropy values were between 1.6 and $5.0 \times 10^{-6}$; the resulting lifetime and intensity values were essentially the same at both entropies for a given dataset. We observe that this routine has not been reported for many instances of positron studies on crystalline molecular samples [28-30], and consider the present results to be preliminary. Two comparative results for probability distribution functions obtained via MELT are shown in Fig. 3. The left part corresponds to the samples in Table I (left), and the right part is a result from the "after" experiments in Table II; both distributions include a very low intensity third component, marked with an asterisk $(*)$. The left part presents 
lifetimes that follow the trend observed in Table I (left), however the values are consistently larger than the PATFIT results by $20-30 \%$. The sample data sets corresponding to Table II gave varied and inconsistent results in MELT analysis. The right part in Fig. 3 illustrates one example. Here, the D-Ala result is fairly consistent with the PATFIT result, but the L-Ala result is significantly higher than the comparable data in Table II. At present, we find the MELT analysis to be intriguing, but not yet to be relied on regarding the alanine single crystals studied here. Additional focused and high quality studies of molecular crystals evaluated by the MELT routine are needed to elaborate on the results presented here [28-30].

\section{Conclusion}

A range of possible stereochemical interactions (stereo-recognition, -selection, or -induction) between polarized particles and asymmetric forms of matter serves as a means of classifying past, present, and future asymmetric experiments involving beta particles. This taxonomy may also serve in the design of new types of experiments to probe asymmetric matter. Asymmetric sets of alanine crystals (L and D forms) from two types of crystallization methods were evaluated by positron annihilation lifetime spectroscopy. The analysis yielded satisfactory results typical for crystalline molecular materials and allowed for the evaluation of possible stereorecognition of chiral single crystals by a positron technique. The neutral result regarding asymmetric interactions may arise from either a loss of asymmetric interaction due to charged functional groups (especially the carboxylate, $\mathrm{COO}^{-}$) or due to a lack of a sufficiently dense asymmetric environment (the crystal packing) for interactions with the positron/Ps species. Future studies will include nonionized, asymmetric amino acids and other asymmetric molecular entities that provide a chiral environment for potential positron/host asymmetric interactions.

\section{Acknowledgments}

The authors thank Bilecik Seyh Edebali University Department of Scientific Research Project Unit for supporting this study as a part of the project 2015-02.BŞEÜ.0402. We thank James Murowchick (UMKC Geosciences) for assistance with crystal handling techniques and optical measurements. The U. S. Department of Energy Office of Science by the Isotope Program in the Office of Nuclear Physics supplied the isotopes used in this project.

\section{References}

[1] A. Guijarro, M. Yus, The Origin of Chirality in the Molecules of Life, RSC Publishing, Cambridge 2009.

[2] W.A. Bonner, Top. Stereochem. 18, 1 (1988).

[3] M. Quack, J. Stohner, M. Willeke, Ann. Rev. Phys. Chem. 59, 741 (2008).
[4] R. Bast, A. Koers, A.S.P. Gomes, M. Ilias, L. Visscher, P. Schwerdtfeger, T. Saue, Phys. Chem. Chem. Phys. 13, 865 (2011).

[5] Y.C. Jean, H.J. Ache, J. Phys. Chem. 81, 1157 (1976).

[6] A.S. Garay, L. Keszthekyi, I. Demeter, P. Hrasko, Nature 250, 332 (1974).

[7] M.M. Ulrich, D.C. Walker, Nature 258, 418 (1975).

[8] A. Rich, Nature 264, 482 (1976).

[9] D.W. Gidley, A. Rich, J. Van House, P.W. Zitzewitz, Nature 297, 639 (1982).

[10] R.A. Hegstrom, Nature 297, 643 (1982).

[11] L. Chiari, A. Zecca, S. Girardi, A. Defant, F. Wang, X.G. Ma, M.V. Perkins, M.J. Brunger, Phys. Rev. A 85, 052711 (2012).

[12] J.M. Dreiling, S.J. Burtwistle, T.J. Gay, Appl. Opt. 54, 763 (2015).

[13] J.M. Dreiling, T.J. Gay, J. Phys. Conf. Ser. 635, 012015 (2015).

[14] J.M. Dreiling, F.W. Lewis, J.D. Mills, T.J. Gay, Phys. Rev. Lett. 116, 093201 (2016).

[15] C. Razzetti, M. Ardoino, L. Zanotti, M. Zha, C. Paorici, Cryst. Res. Technol. 37, 456 (2002).

[16] W. Wang, F. Yi, Y. Ni, Z. Zhao, X. Jin, Y. Tang, J. Biol. Phys. 26, 51 (2000).

[17] Y.C. Jean, J.D. Van Horn, W.-S. Hung, K.-R. Lee, Macromolecules 46, 7133 (2013).

[18] Z. Xia, M. Trexler, F. Wu, Y.C. Jean, J.D. Van Horn, Phys. Rev. E 89, 022603 (2014).

[19] M.B. Larsen, J.D. Van Horn, F. Wu, M.A. Hillmyer, Macromolecules 50, 4363 (2017).

[20] X. Hong, Y.C. Jean, H. Yang, S.S. Jordan, W.J. Koros, Macromolecules 29, 7859 (1996).

[21] P. Kirkegaard, M. Eldrup, O.E. Mogensen, N.J. Pedersen, Comput. Phys. Commun. 23, 307 (1981).

[22] A. Shukla, M. Peter, L. Hoffmann, Nucl. Instrum. Methods Phys. Res. A 335, 310 (1993).

[23] Chirality: Physical Chemistry, Ed. J.M. Hicks, Am. Chem. Soc., Washington, DC 2002.

[24] P.W. Zitzewitz, J.C. Van House, A. Rich, D.W. Gidley, Phys. Rev. Lett. 43, 1281 (1979).

[25] J. Van House, P.W. Zitzewitz, Phys. Rev. A 29, 96 (1984).

[26] J.D. Van Horn, F. Wu, G. Corsiglia, Y.C. Jean, Defect. Diffus. Forum 373, 221 (2016).

[27] O. Czupiński, A. Ingram, M. Kostrzewa, J. Przesławski, Z. Czapla, Acta Phys. Pol. A 131, 304 (2017)

[28] M. Tydda, B. Jasińska, Nukleonika 60, 771 (2015).

[29] B. Jasińska, A.E. Kozioł, T. Goworek, Acta Phys. Pol. A 95, 557 (1999).

[30] M. Eldrup, D. Lightbody, J.N. Sherwood, Chem. Phys. 63, 51 (1981). 